\title{
Exploring the Young People's Cognitive Structure and Switching Intention Toward Social Networking Sites: An Abstract
}

\author{
Chin-Feng Lin and Chen-Su Fu
}

\begin{abstract}
This study, based on means-end chains (MECs) and push-pull-mooring (PPM) model, aims to reveal the functional attributes of Facebook (FB) and Instagram (IG), classify them into PPM model (i.e., push, pull, and mooring effect), and then integrate the nature of MECs (i.e., attribute-consequence-value linkages) to analyze young people's perceptions toward FB and IG and their switching intention. By integrating MEC results and PPM model, a hybrid hierarchical value map (HVM) was constructed to provide a complete picture of FB/IG users' switching intention. The hybrid HVM shows that "hiding posts from someone" and "privacy settings" (push effects) are the unfavorable attributes of FB to push FB users to migrate to IG, while "direct function" and "tag' function" (pull effects) are the favorable attributes of IG to attract FB users to migrate. Moreover, the "photo filter" and "tracking public figures or fan pages" (mooring effect) are the attributes that facilitate or impede FB users to migrate. By understanding the hybrid HVM, it is possible to provide designers with valuable insights for FB/IG design and improvement, so the managers and designers of social networking sites are able to develop their effective business strategies.
\end{abstract}

\footnotetext{
C.-F. Lin $(\triangle)$

National Pingtung University, Pingtung City, Taiwan

e-mail: cfl@mail.nptu.edu.tw

C.-S. Fu

National Cheng Kung University, Tainan City, Taiwan

e-mail: csfu1223@gmail.com
} 